\title{
Residual Stresses in Glasses
}

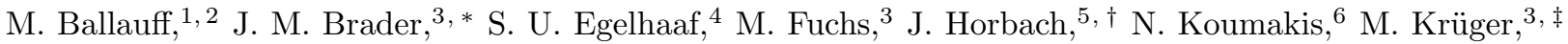 \\ M. Laurati, ${ }^{4}$ K. J. Mutch, ${ }^{4}$ G. Petekidis, ${ }^{6}$ M. Siebenbürger, ${ }^{1,2}$ Th. Voigtmann, ${ }^{3,5,7}$ and J. Zausch ${ }^{8, \$}$ \\ ${ }^{1}$ Soft Matter and Functional Materials, Helmholtz-Zentrum für Materialien und Energie, 14109 Berlin, Germany \\ ${ }^{2}$ Institut für Physik, Humboldt-Universität zu Berlin, 10099 Berlin, Germany \\ ${ }^{3}$ Fachbereich Physik, Universität Konstanz, 78457 Konstanz, Germany \\ ${ }^{4}$ Soft Matter Laboratory, IPKM, Heinrich-Heine Universität Düsseldorf, Universitätsstr. 1, 40225 Düsseldorf, Germany \\ ${ }^{5}$ Institut für Materialphysik im Weltraum, Deutsches Zentrum für Luft- und Raumfahrt (DLR), 51170 Köln, Germany \\ ${ }^{6}$ IESL-FORTH and Department of Materials Science and Technology, University of Crete, Heraklion 71110, Crete, Greece \\ ${ }^{7}$ Zukunftskolleg, Universität Konstanz, 78457 Konstanz, Germany \\ ${ }^{8}$ Institut für Physik, Johannes-Gutenberg-Universität Mainz, Staudinger Weg 7, 55099 Mainz, Germany
}

(Dated: November 18, 2018)

\begin{abstract}
The history dependence of the glasses formed from flow-melted steady states by a sudden cessation of the shear rate $\dot{\gamma}$ is studied in colloidal suspensions, by molecular dynamics simulations, and modecoupling theory. In an ideal glass, stresses relax only partially, leaving behind a finite persistent residual stress. For intermediate times, relaxation curves scale as a function of $\dot{\gamma} t$, even though no flow is present. The macroscopic stress evolution is connected to a length scale of residual liquefaction displayed by microscopic mean-squared displacements. The theory describes this history dependence of glasses sharing the same thermodynamic state variables, but differing static properties.

PACS numbers: 64.70.P- 83.50.-v
\end{abstract}

Materials are often produced by solidification from the melt, involving nonequilibrium quenches. This imprints a history-dependent microstructure that strongly affects macroscopic material properties. One example is residual stresses [1, 2]: if particle configurations cannot fully relax to equilibrium, some of the stresses, arising in the presence of flow in the melt, persist in the solid.

Small glass droplets (known as Prince Rupert's drops or Dutch tears since the 17 th century) vividly display the effects of residual stresses 3: they withstand the blow of a hammer onto their main body, but explode when the slightest damage is inflicted upon their tail (releasing the frozen-in stress network). Today, safety glass and "Gorilla glass" covers for smartphones are deliberately pre-stressed during production to strengthen them. A theoretical understanding of residual stresses and their microscopic origins is however still not achieved.

We seek to understand generic mechanisms by which residual stresses arise. A convenient starting point is to investigate the stress relaxation $\sigma(t)$ following the cessation of shear flow of rate $\dot{\gamma}$, from a well-defined nonequilibrium stationary state (NESS). Such "mechanical quenches" are ubiquitous in soft matter, where pre-shear is applied to "rejuvenate" the otherwise ill-defined glassy state [4 7]. For these systems, the soft-glassy rheology model (SGR) 8 predicts asymptotic power laws that imply the relaxation of stresses to zero [9]. In the following, we will reserve the term residual stress to describe a finite, persistent stress remaining in the (ideal) glass even at arbitrarily large times after the cessation of flow.

In addition to macroscopic rheology, we investigate the evolution of the microscopic dynamics as characterized by the waiting-time dependent mean-squared displacements (MSD). The latter reveal the dynamical shrinkage of shear-fluidized regions after cessation, and phenomena akin to, yet different from the intensely studied aging dynamics after thermal quenches [10, 11].

Experiments on a variety of colloidal suspensions, together with molecular-dynamics (MD) simulations, provide a coherent qualitative picture that can be rationalized by mode-coupling theory of the glass transition (MCT) [12] within the integration-through-transients (ITT) formalism [13. The theory in particular predicts the existence of a residual stress in the glass, the magnitude of which depends on the history of shear. We expect the same mechanisms to be generically valid for many colloidal as well as molecular - glass formers.

The experiments, theory, and simulation are described in detail in the Supplementary Information (SI) 14] and summarized here. We perform rheology on colloidal polystyrene-PNIPAM core-shell particles in aqueous solution (PP) [15, 16, and on PMMA particles in different solvents (HS) [17 19]. These are well studied, nearly hardsphere glass formers 20, but differ in particle properties like softness and polydispersity. The average size is $R \approx 90 \mathrm{~nm}(\mathrm{PP})$ and $R \approx 267 \mathrm{~nm}$ (HS) for macroscopic rheology, and $R \approx 770 \mathrm{~nm}$ (HS) for confocal microscopy. Density is expressed as a dimensionless packing fraction $\varphi$; the glass transition occurs in the two systems at $\varphi_{c} \approx 0.64(\mathrm{PP})$ and $\varphi_{c} \approx 0.59(\mathrm{HS})$. In the PP system, the effective packing fraction is sensitively tuned through temperature.

MD simulations using a dissipative-particle-dynamics thermostat were performed on a binary glass-forming Yukawa mixture as outlined in Refs. [19, 21]. The unit of length was chosen as the small-particle radius $R$. The glass transition temperature of this system is $T_{c} \approx 0.14$.

ITT-MCT was evaluated numerically 22, 24, with 


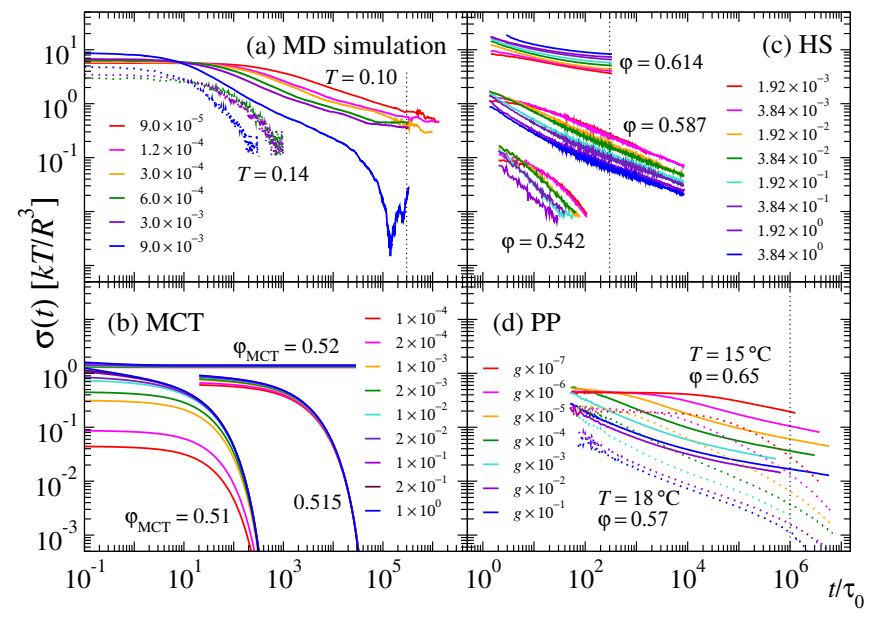

FIG. 1. Stress decay $\sigma(t)$ after cessation of steady shear (time in units of the microscopic relaxation scale $\tau_{0}$ ), for various shear rates $\dot{\gamma} \tau_{0}=P e_{0}$ (increasing from red to blue) and control parameters, as labeled. Dotted lines indicate times used to extract residual stresses in Fig. 2 (a) MD simulation: $T=0.14$ in the liquid, $T=0.1$ in the glass. (b) Isotropic hard-sphere model of ITT-MCT, $\varphi_{\mathrm{MCT}}=0.51,0.515$ (liquid), and 0.52 (glass). (c) HS colloidal suspension: $\varphi=0.542$, 0.587 (liquid), and 0.614 (glass). (d) PS-PNIPAM particles: $T=18{ }^{\circ} \mathrm{C}(\varphi \approx 0.57$, liquid, $\dot{\gamma}$ as labeled with $g=3.4)$ and $T=15^{\circ} \mathrm{C}(\varphi \approx 0.65$, glass, $g=4.0)$.

the Percus-Yevick model of the equilibrium hard-sphere structure (giving $\varphi_{c} \approx 0.516$ ) and an isotropic approximation to spatial integrals. This model (ISHSS) has been used together with our MD system and confocal microscopy to study the evolution from equilibrium to the NESS [19, 25. Mean-squared displacements are calculated using a schematic model based on Refs. [26, 27].

Figure 1 shows the transient decay of the shear stress $\sigma(t)$ measured in rheology and computer simulation, and calculated within ITT-MCT. For each system, curves for various shear rates $\dot{\gamma}$, and thermodynamic control variables above and below the glass transition are shown. Stresses are reported in entropic units, $k T / R^{3} \approx 0.21 \mathrm{~Pa}$ (HS), 5.33 Pa and $5.82 \mathrm{~Pa}\left(\mathrm{PP}\right.$ at $T=15^{\circ} \mathrm{C}$ and $\left.18^{\circ} \mathrm{C}\right)$. Values measured in the two colloidal suspensions differ by a factor 25 in their absolute value, consistent with their size difference. Such scale differences do not change the qualitative rheology of dense liquids where structural relaxation governs the dynamics 28. The shear rate $\dot{\gamma}$ is switched off at $t=0$ after all systems have reached a welldefined NESS, imposing a constant strain for all $t>0$. Times are reported relative to the scale of single-particle motion; related to free diffusion $D_{0}, \tau_{0}=R^{2} / D_{0} \approx 0.3 \mathrm{~s}$ (HS), $4.0 \mathrm{~ms}$ and $3.4 \mathrm{~ms}(\mathrm{PP})$, or to ballistic motion and the potential energy scale $\epsilon, \tau_{0}=\sqrt{4 m R^{2} / \epsilon}(\mathrm{MD})$. (The different forms of short-time motion may result in a shift of the relevant $t / \tau_{0}$ when comparing features of the longtime dynamics [29, 30].) Curves start from the corresponding steady-state value $\sigma(t=0)=\sigma_{\text {ss }}(\dot{\gamma})$ (separately

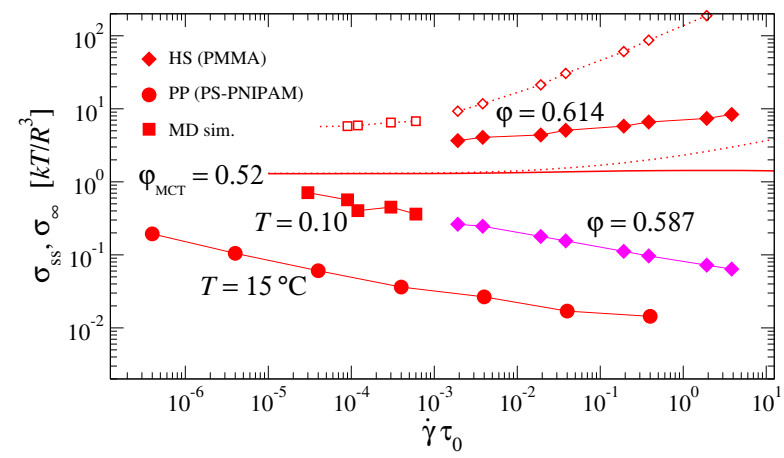

FIG. 2. Steady-state flow curve $\sigma_{\mathrm{ss}}(\dot{\gamma})$ (open symbols) and residual stress $\sigma_{\infty}(\dot{\gamma})$ (filled) determined from experiment as $\sigma_{\infty} \approx \sigma\left(t_{\infty}\right)$ in Fig. 1 (diamonds: HS, circles: PP) and MD simulation (squares). A solid (dotted) line shows the residual stress (flow curve) for the MCT model of Fig. 1

measured), which is a nonlinear function of the shear rate (called the flow curve).

Stresses in dense liquids are dominated by the structural contribution, hence slow glassy dynamics governs their decay at long times. In the fluid, $\sigma(t)$ relaxes to zero on the structural-relaxation time scale $\tau$. Approaching the glass transition, $\tau$ grows beyond the experimental window, and intermediate plateaus develop in the stress relaxation. In the MCT idealization, $\tau$ diverges as permanent local caging of particles prevents the full relaxation of density fluctuations. The previously shearmolten ideal glass is then characterized by a non-relaxing persistent residual stress $\sigma_{\infty}(\dot{\gamma})=\lim _{t \rightarrow \infty} \sigma(t)>0$. Its shear-rate dependence highlights the nonequilibrium nature of the glassy state attained after imposing zero-flow conditions: different glasses exist with the same thermodynamic control parameters, but different historydependent frozen-in properties.

In simulation and experiment, the residual-stress plateaus slowly decay, possibly as a result of creep [3133, which is precluded in the present MCT calculations. Imposing zero-stress instead of zero-strain-rate conditions on the other hand will allow for rejuvenation effects that may eliminate residual stresses [32, 33].

The residual stress $\sigma_{\infty}(\dot{\gamma})$ as a function of the pre-shear rate is shown in Fig.2(filled symbols). In experiment and simulation, comparable values have been determined at a suitable intermediate time $t_{\infty}$ (marked in Fig. 1). Also shown (open symbols) are some of the flow curves $\sigma_{\text {ss }}(\dot{\gamma})$. They exhibit two features typical for glass-forming fluids: a dynamical yield stress, $\sigma_{y}=\lim _{\dot{\gamma} \rightarrow 0} \sigma_{\mathrm{ss}}(\dot{\gamma})>0$, and a monotonic increase with increasing $\dot{\gamma}$. The residual stresses $\sigma_{\infty}(\dot{\gamma})$, on the other hand, typically decrease with increasing $\dot{\gamma}$ (leading to a crossing of $\sigma(t)$-versus- $t$ curves at short $t / \tau_{0}$, see Fig. 1 1 ): the stronger the past fluidization (and hence, structural distortions [18]) of the glass, the more effective the stress relaxation.

For $\dot{\gamma} \rightarrow 0$, the residual stress $\sigma_{\infty}$ approaches $\sigma_{y}$. In 


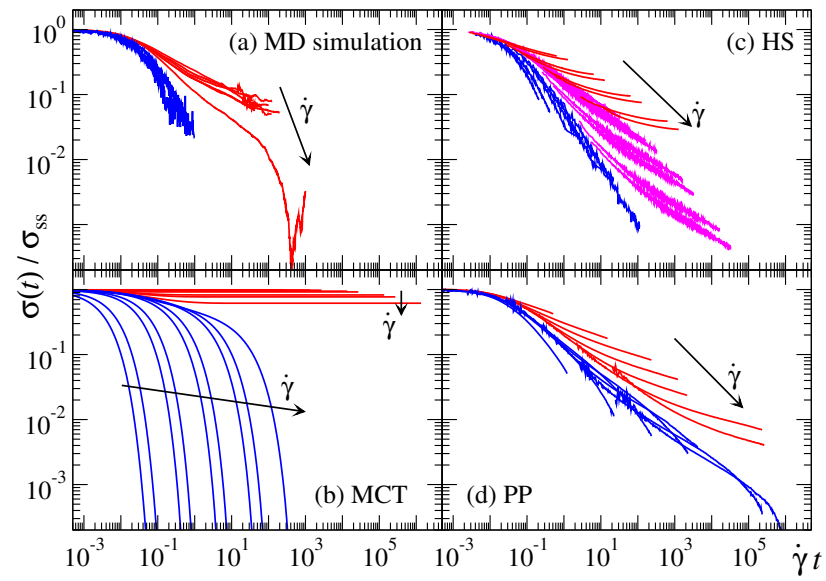

FIG. 3. As Fig. 1 but $\sigma(t) / \sigma_{\text {ss }}$ as a function of $\dot{\gamma} t$. Glass (liquid) states are shown in red (blue).

ITT-MCT, the two quantities coincide in this limit, implying that for arbitrarily slow flow, the glass attains a certain stress that can never relax, even after the perturbation is removed. To understand this, recall that the shear stress is given by a nonlinear Green-Kubo relation, $\sigma_{x y}(t)=\int_{-\infty}^{t} \dot{\gamma}\left(t^{\prime}\right) G\left(t, t^{\prime},[\dot{\gamma}]\right) d t^{\prime}$, where the generalized shear modulus $G\left(t, t^{\prime},[\dot{\gamma}]\right)$ is a transient correlation function that is formed with the equilibrium ensemble average and is only affected by external perturbations active between its two time arguments $t^{\prime}<t[23$. In the absence of other relaxation mechanisms, the same physical process, shear-induced breaking of cages on a time scale $\tau_{\dot{\gamma}} \sim 1 / \dot{\gamma}$, dominates the history integrals determining $\sigma_{y}$ and $\sigma_{\infty}$, and $\sigma_{\infty} \approx \sigma_{y}>0$ results. The ISHSS model of ITT-MCT predicts a slight increase of $\sigma_{\infty}$ with increasing $\dot{\gamma}$, which is only seen in the HS experiment well in the glass.

The stress relaxations reveal remarkable scaling behavior close to the glass transition. Normalizing stresses by their flow-curve value, and rescaling time with the initial shear rate, the relaxation curves fall into two classes as shown in Fig. 3. Two distinct decay patterns - one for the liquid, one for the glass - emerge that provide a clear indicator to locate the glass transition through a series of shear-cessation experiments. The dependence on "pseudo-strain" $\dot{\gamma} t$, as if flow persisted, is remarkable since the equations of motion at $t>0$ contain no reference to the past perturbation. The scaling hence can be thought of as the slow decay of the NESS-contributions to the distribution function over which dynamical quantities are averaged. In contrast, the final decay of $\sigma(t)$ to zero in the liquid does not scale with $\dot{\gamma}$, as it is governed by the equilibrium relaxation time.

The SGR predicts the observed scaling with $\dot{\gamma} t$ based on aging phenomena 9. Specifically, one obtains an asymptotic power-law decay to zero, $\sigma(t) / \sigma_{\mathrm{ss}} \sim(\dot{\gamma} t)^{-x}$ for $\dot{\gamma} t \gg 1$, where $x$ is a temperature-like parameter. A

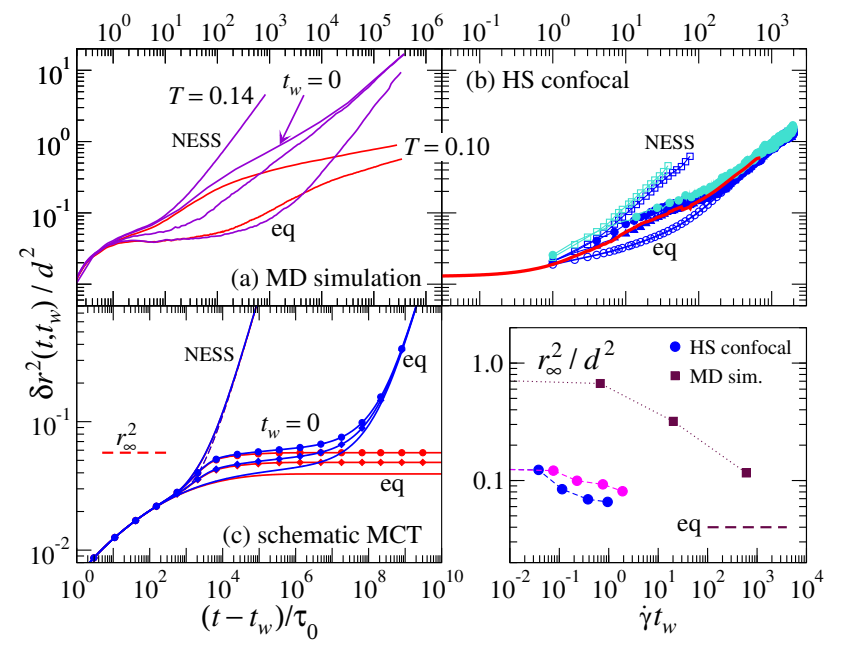

FIG. 4. Mean-squared displacement $\delta r^{2}\left(t, t_{w}\right)$ starting at a waiting time $t_{w}$ after cessation of steady shear, in units of particle diameters. (a) MD simulation (large particles): for $T=0.14, \dot{\gamma} t_{w}=0$ and 0.675 for $\dot{\gamma} \tau_{0}=3 \times 10^{-3}$ together with the NESS and equilibrium result (violet). For $T=0.1$, $t_{w}=0$ curves for $\dot{\gamma} \tau_{0}=3 \times 10^{-3}$ and $3 \times 10^{-5}$ (red; right to left). (b) Confocal microscopy on hard-sphere PMMA colloids, at $\varphi=0.56$. Curves for NESS and equilibrium together with $t_{w}=0$ for $\dot{\gamma} \tau_{0}=0.0038 / \mathrm{s}$ and $0.0076 / \mathrm{s}\left(P e_{0} \approx 0.0097\right.$ and 0.019$)$. For the smaller shear rate, $t_{w}=4 \mathrm{~s}$ is also shown. The thick red line is a theory fit to this curve. (c) Schematic ITT-MCT model, see text, for two state points $\varepsilon= \pm 10^{-4}$ (glass/liquid) with $\dot{\gamma} \tau_{0}=10^{-5}$, and $t_{w}=0$ and $\dot{\gamma} t_{w}=0.01$. (d) Intermediate-plateau values after switch-off, $r_{\infty}^{2}$, as a function of $\dot{\gamma} t_{w}$. A dashed line marks the equilibrium plateau height from MD.

finite residual stress $\sigma_{\infty}$ is not predicted by the SGR.

To elucidate the microscopic mechanisms at play during stress relaxation, we turn to the mean-squared displacements (MSD) of individual particles. This is a twotime average, $\delta r^{2}\left(t, t_{w}\right)=\left\langle\left(\vec{r}(t)-\vec{r}\left(t_{w}\right)\right)^{2}\right\rangle_{t_{w}}$, governed by the equilibrium dynamics but averaged with respect to the statistical ensemble at a waiting time $t_{w}>0$ after cessation of shear. MSD in the vorticity direction, for various shear rates and state points, and for various $t_{w}$, from confocal-microscopy experiments and MD simulations are shown in Fig. 4. The $t_{w}$-independent MSD measured in the equilibrium fluid and in the sheared NESS are shown as a reference. They exhibit an intermediatetime plateau indicating transient caging of particles in their nearest-neighbor shells. Long-time diffusion is enhanced in the NESS, a non-linear response effect that is the microscopic analogue of shear thinning [34. Measuring the MSD with reference to the configuration at the cessation point, $t_{w}=0$, the dynamics still follows that of the NESS, up to a time given by $\dot{\gamma} t \approx 0.1$. This connects to a typical strain that causes cage-breaking [19]. The MSD then slowly crosses over to the equilibrium dynamics, indicating an intermediate subdiffusive plateau that is larger than the one connected to quiescent caging. At 
times $t-t_{w} \gtrsim \tau$, all curves become independent of $t_{w}$ and collapse on the equilibrium curves. For MD simulations in the glassy state the intermediate plateau extends past the simulated time window. The height of this plateau decreases with increasing $\dot{\gamma}$.

ITT-MCT (extended to describe waiting-time dependent two-time averages for small $t_{w}$ [14, 26, 27]) qualitatively rationalizes these MSD. Figure 4 includes schematic-model results for both a glassy and a liquid state. A well-defined second plateau emerges in the crossover from the NESS to equilibrium curves and becomes permanent in the glass. It can be interpreted as a second length scale beyond the quiescent localization length, arising from the competition of shear-induced fluidization and arrest after cessation. Intuitively, it is a "Bremsweg" (stopping distance) for individual particles caused by the progression of the distribution function from the perturbed state at $t_{w}$ to the quiescent state. But note that inertial effects play no role in the dynamics and are, by construction, absent from the theory.

The ITT-MCT model approximates the $t_{w}$-dependent MSD as a combination of the equilibrium and NESS ones. Given this information from experiments, the evolution of $\delta r^{2}\left(t, t_{w}\right)$ for small $t_{w}$ can be predicted. This is demonstrated by the fit to the HS data shown in Fig. $4 \mathrm{~b}$.

The scenario of nonequilibrium relaxation discussed here characteristically differs from aging dynamics in spin glasses [35, 36] or that following density quenches in hard spheres $37+39$. There the structural relaxation time $\tau\left(t_{w}\right)$ grows with sample age, so that correlation functions and related two-time averages depend on $t_{w}$ in their long-time part, while the short-time relaxation for increasing sample age reveals more and more of an intrinsic, $t_{w}$-independent relaxation. For the MSD, this implies a continuous shift of the long-time diffusive asymptote (where $\delta r^{2} \sim t$ ) to longer times. In contrast, in Fig. 4 all intermediate- $t_{w}$ curves show subdiffusive transients and an approach to the same $t_{w}$-independent long-time diffusion found in the quiescent system.

In conclusion, we have studied stress decays and the microscopic dynamics of glass-forming liquids and shearmelted glasses after the cessation of steady shear flow. In the liquid, stresses relax to zero following long transients, on the time scale of the quiescent equilibrium system. (In rheological terms, the systems exhibit "thixotropy".)

Finite residual stresses remain in the glass. Their value and the initial evolution from the steady state to the nonequilibrium quiescent solid, are governed by the pre-shear rate through long-lived memory effects. These memory effects cause the appearance of a microscopic supra-caging length scale in the waiting-time dependent mean-squared displacements.

Residual stresses imply that glass is not simply characterized by its thermodynamic control variables. Different preparation histories result in glasses that differ subtly in their structure, and possibly also in their response behavior, for example their elastic moduli 4. Within ITT-MCT, a pre-strain dependence of the shear modulus has been studied in the flowing steady state [40]. It would be enlightening to compare this to a history dependence that arises in the case of temperature quenches, as recently studied in computer simulations [41.

The theory describes history-dependent glass states through retarded-friction contributions to the dynamics of density fluctuations that are modified by the past flow. This goes beyond traditional near-equilibrium glass transition theories (such as standard MCT), where the relaxation of small initial perturbations induced by an external field is related to a Kubo correlator of equilibrium fluctuations. This connection is based on Onsager's regression hypothesis, and it holds if the initial perturbation obeys equilibrium linear-response theory. It is violated in the glass (unless activated processes restore ergodicity) 42, since infinitesimally small rates shear-melt the glass [13]. These states relax only due to external driving, which is the common cause of both a dynamical yield stress $\sigma_{y}=\lim _{\dot{\gamma} \rightarrow 0} \sigma_{\mathrm{ss}}(\dot{\gamma})>0$ (contradicting linear response), and of a residual stress $\sigma_{\infty}$ (contradicting equilibrium).

The qualitative agreement among different systems and methods suggests that our discussion applies generally to glass formers where excluded volume effects dominate, causing stresses to be given by the entropic scale $k T / R^{3}$. This includes dense metallic glass formers, where an understanding of frozen-in residual stresses is important for understanding material stability.

In such systems, possibly also for colloidal systems deep in the glass, additional effects may need to be accounted for, connected to spatial and temporal flow heterogeneities. Then, concepts such as sheartransformation zones (STZ) 43] or stress avalanches [44, 45] (or, in the case of granular materials, force chains 46]) can become important. For the data presented above, we have checked that no such inhomogeneities are detectable either in confocal microscopy or during the simulation runs.

Th. V. is funded by the Helmholtz Gesellschaft (HGF, VH-NG 406), and Zukunftskolleg der Universität Konstanz; M. K. by DFG grant KR 3844/2-1; G. P. and N. K. by Thales Project "Covisco" and EU project "ESMI". We thank for funding through DFG FOR 1394, project P3, and SFB-TR6, projects A5 and A6.

* Present address: Department of Physics, University of Fribourg, CH-1700 Fribourg, Switzerland

† Present address: Soft Matter Laboratory, IPKM, Heinrich-Heine Universität Düsseldorf, Universitätsstr. 1, 40225 Düsseldorf, Germany

¥ Present address: Institute for Theoretical Physics IV, Universität Stuttgart, Pfaffenwaldring 57, 70569 
Stuttgart, and Max-Planck-Institut für Intelligente Systeme, Heisenbergstraße 3, 70569 Stuttgart, Germany

$\S$ Present address: Fraunhofer-Institut für Techno- und Wirtschaftsmathematik, Abteilung Strömungs- und Materialsimulation, Fraunhofer-Platz 1, 67663 Kaiserslautern, Germany

[1] P. J. Withers, Rep. Prog. Phys. 70, 2211 (2007).

[2] G. Reiter, M. Hamieh, P. Damman, S. Sclavons, S. Gabriele, T. Vilmin, and E. Raphaël, Nature Mater. 4, 754 (2005).

[3] L. Brodsley, C. Frank, and J. W. Steeds, Notes Rec. R. Soc. Lond. 41, 1 (1986).

[4] P. Moldenaers and J. Mewis, J. Rheol. (NY) 30, 567 (1986).

[5] V. Viasnoff and F. Lequeux, Phys. Rev. Lett. 89, 065701 (2002).

[6] A. S. Negi and C. O. Osuji, J. Rheol. (NY) 54, 943 (2010).

[7] G. Yin and M. J. Solomon, J. Rheol. (NY) 52, 785 (2008).

[8] P. Sollich, F. Lequeux, P. Hébraud, and M. E. Cates, Phys. Rev. Lett. 78, 2020 (1997).

[9] M. E. Cates and P. Sollich, J. Rheol. (NY) 48, 193 (2004).

[10] L. C. E. Struik, Physical Aging in Amorphous Polymers and Other Materials (Elsevier, Amsterdam, 1978).

[11] J.-L. Barrat, J. Phys.: Condens. Matter 15, S1 (2003).

[12] W. Götze, Complex Dynamics of Glass-Forming Liquids (Oxford University Press, Oxford, 2009).

[13] M. Fuchs and M. E. Cates, Phys. Rev. Lett. 89, 248304 (2002).

[14] See EPAPS Document No. E-PRLTAO000-000000 for supplementary material. For more information on EPAPS, see http://www . aip.org/pubservs/epaps.html.

[15] N. Dingenouts, Ch. Norhausen, and M. Ballauff, Macromolecules 31, 8912 (1998).

[16] M. Siebenbürger, M. Fuchs, H. Winter, and M. Ballauff, J. Rheol. (NY) 53, 707 (2009).

[17] N. Koumakis, A. B. Schofield, and G. Petekidis, Soft Matter 4, 2008 (2008).

[18] N. Koumakis, M. Laurati, S. U. Egelhaaf, J. F. Brady, and G. Petekidis, Phys. Rev. Lett. 108, 098303 (2012).

[19] J. Zausch, J. Horbach, M. Laurati, S. U. Egelhaaf, J. M. Brader, Th. Voigtmann, and M. Fuchs, J. Phys.: Condens. Matter 20, 404210 (2008).

[20] N. Koumakis, A. Pamvouxoglou, A. S. Poulos, and G. Petekidis, Soft Matter 8, 4271 (2012).

[21] J. Zausch and J. Horbach, EPL 88, 60001 (2009).

[22] M. Fuchs and M. E. Cates, J. Rheol. (NY) 53, 957 (2009).

[23] J. M. Brader, Th. Voigtmann, M. Fuchs, R. G. Larson, and M. E. Cates, Proc. Natl. Acad. Sci. (USA) 106, 15186 (2009).

[24] Th. Voigtmann, J. M. Brader, M. Fuchs, and M. E. Cates, Soft Matter 8, 4244 (2012).

[25] M. Laurati, K. J. Mutch, N. Koumakis, J. Zausch, C. P. Amann, A. B. Schofield, G. Petekidis, J. F. Brady, J. Horbach, M. Fuchs, and S. U. Egelhaaf, J. Phys.: Condens.
Matter 24, 464104 (2012).

[26] M. Krüger and M. Fuchs, Phys. Rev. Lett. 102, 135701 (2009).

[27] M. Krüger, F. Weysser, and Th. Voigtmann, Phys. Rev. E 81, 061506 (2010).

[28] C. Amann, F. Weysser, M. Krüger, S. Fritschi, M. Siebenbürger, M. Ballauff, K. Samwer, M. Laurati, S. U. Egelhaaf, Th. Voigtmann, and M. Fuchs, (2013), submitted.

[29] T. Gleim, W. Kob, and K. Binder, Phys. Rev. Lett. 81, 4404 (1998).

[30] Th. Voigtmann, A. M. Puertas, and M. Fuchs, Phys. Rev. E 70, 061506 (2004).

[31] M. Siebenbürger, M. Ballauff, and Th. Voigtmann, Phys. Rev. Lett. 108, 255701 (2012).

[32] G. Petekidis, D. Vlassopoulos, and P. N. Pusey, Faraday Discuss. 123, 287 (2003).

[33] G. Petekidis, D. Vlassopoulos, and P. N. Pusey, J. Phys.: Condens. Matter 16, S3955 (2004).

[34] R. Besseling, E. R. Weeks, A. B. Schofield, and W. C. K. Poon, Phys. Rev. Lett. 99, 028301 (2007).

[35] F. Belletti, M. Cotallo, A. Cruz, L. A. Fernandez, A. Gordillo-Guerrero, M. Guidetti, A. Maiorano, F. Mantovani, E. Marinari, V. Martin-Mayor, A. Muñoz Sudupe, D. Navarro, G. Parisi, S. Perez-Gaviro, J. J. RuizLorenzo, S. F. Schifano, D. Sciretti, A. Tarancon, R. Tripiccione, J. L. Velasco, and D. Yllanes, Phys. Rev. Lett. 101, 157201 (2008).

[36] R. Mathieu, M. Hudl, and P. Nordblad, EPL 90, 67003 (2010).

[37] A. M. Puertas, J. Phys.: Condens. Matter 22, 104121 (2010).

[38] G. Pérez-Ángel, L. E. Sánchez-Díaz, P. E. RamírezGonzález, R. Juárez-Maldonado, A. Vizcarra-Rendón, and M. Medina-Moyola, Phys. Rev. E 83, 060501(R) (2011).

[39] X. Di, K. Z. Win, G. B. McKenna, T. Nartia, F. Lequeux, S. R. Pullela, and Z. Cheng, Phys. Rev. Lett. 106, 095701 (2011)

[40] F. Frahsa, A. K. Bhattacharjee, J. Horbach, M. Fuchs, and Th. Voigtmann, J. Chem. Phys. 138, 12A513 (2013).

[41] Ashwin J., E. Bouchbinder, and I. Procaccia, "The cooling rate dependence of the shear modulus of amorphous solids," (2013), arXiv:1301.4664.

[42] S. R. Williams and D. J. Evans, Phys. Rev. Lett. 96, 015701 (2006).

[43] M. L. Falk and J. S. Langer, Annu. Rev. Cond. Matt. Phys. 2, 353 (2011).

[44] A. Lemaître and C. Caroli, Phys. Rev. Lett. 103, 065501 (2009).

[45] J. Chattoraj, C. Caroli, and A. Lemaître, Phys. Rev. E 84, 011501 (2011).

[46] R. R. Hartley and R. P. Behringer, Nature 421, 928 (2003). 